\title{
Virtual Wind Tunnel: an efficient computational tool for aeroelastic analysis and design of inflatable structures
}

\author{
Jose Manuel GONZALEZ ${ }^{\mathrm{a}}$, Enrique ORTEGA ${ }^{\mathrm{b}, \mathrm{a}}$, Jordi PONS-PRATS ${ }^{\mathrm{c}, \mathrm{a}}$, Mercè LÓPEZ ${ }^{\mathrm{a}}$, Roberto \\ FLORES $^{\mathrm{a}, \mathrm{d}}$, Javier MARCIPAR ${ }^{\mathrm{e}}$, Eugenio OÑATE ${ }^{\mathrm{a}}$ \\ ${ }^{\text {a }}$ International Center for Numerical Methods in Engineering (CIMNE) \\ ${ }^{\mathrm{b}}$ Serra Hunter Professor at Escola Superior d'Enginyeries Industrials, Aeroespacial i Audiovisual de Terrassa \\ (ESEIAAT) Universitat Politècnica de Catalunya, Spain \\ c Serra Hunter Professor at Escola d'Enginyeries de Telecomunicacions i Aeroespacial de Castelldefels \\ (EETAC) Universitat Politècnica de Catalunya, Spain \\ d Aerospace Engineering Department, Khalifa University, Abu Dhabi, UAE \\ e BuildAir - Ingeniería y Arquitectura
}

\begin{abstract}
Buildair Virtual Wind Tunnel (VWT) is an analysis software under development at CIMNE in collaboration with Buildair S.A. to improve the modeling of inflatable structures. This new tool is designed for efficiency, enabling realistic Fluid-Structure Interaction (FSI) simulations with limited computational cost and short turnaround time. Buidair VWT delivers improved accuracy compared to traditional quasi-static estimated solutions with standardized wind pressures, which tend to overestimate loads. This is achieved without resorting to expensive high-fidelity CFD solutions, which are impractical given the resources available to small and medium-sized companies.
\end{abstract}

Buildair VWT combines potential flow aerodynamics, explicit FE structural dynamics and staggered FSI coupling. The aerodynamic solver is a low-order panel method with empirical corrections to account for flow separation effects. In a first step, the detached flow areas are predicted using Stratford's method. Next, the computed pressure in the wake region is adjusted with an ad-hoc model based on the observed behavior of high-Reynolds flows around bluff bodies. Standard wind profiles can be defined to account for the effect of atmospheric boundary layers. On the structural side, the solver uses a large-displacement Finite Element discretization that models cable, membranes and 3D solids using 2-node linear, 3-node triangular and 4-node tetrahedral elements. A wrinkling formulation allows for buckling under compressive loads. The discrete equations are solved in time with an explicit second-order centered scheme. Although conditionally stable, the method is robust and efficient when applied to highly nonlinear problems. Numerical dissipation, including Rayleigh damping and bulk viscosity, is used to control local high-frequency modes that are not well resolved with staggered coupling, and to accelerate the converge to steady-state in static problems. The structural model includes other important loading cases, such as snow accumulation, deflation and general time-varying point and distributed loads defined by the user.

In Buildair VWT special attention has been given to the graphical user interface. It is based on CIMNE's pre and post-processing system GiD, and is designed for ease of use and to minimize the time required for the problem setup, solution and analysis of results. The geometric model can be constructed entirely inside GiD, using the built-in CAD tools geometry generation and manipulation, or imported fromstandard CAD formats. A module for automatic model generation is also available. This allows faster analysis of typical hangar structures and simplifying sensitivity analyses involving geometric and operational parameters. Finally, the post-process tools offer advanced solution analysis and visualization 
13 - 15 September 2021, Munich, Germany

capabilities. The simulation results can also be exported to third-party visualization packages. It is worth mentioning that the structural response to wind loads is typically unsteady. The unsteady results often require averaging before they can be used for design purposes. To this end, Buildair VWT includes a filtering module that considers the relevant range of frequencies and also identifies spurious results. Statistical methods are used to determine the relevant values of deformation, stresses and reaction forces to be used in structural sizing.

The software is currently undergoing extensive testing and validation. Early comparisons with current analysis practices at the company validated the quality of the results and highlighted the importance of including aeroelastic effects in the design of inflatable structures. Incorporating Buildair VWT to the product design and maintenance cycle will provide improved guidance for structural optimization. This will reduce manufacturing costs and facilitate transport and installation. It will also expand the operational envelope to scenarios where standard analysis techniques are unreliable.

Buildair VWT is built around a user-friendly graphical interface. The package documentation includes a theory manual describing the mathematical formulation, as well as a user's manual to help get the most out of the software with minimal training requirements. The structure of the computer program is versatile. It can be easily adapted to problems in the fields of civil, wind and aeronautical engineering.

Keywords: inflatable structures, fluid-structure interaction, panel methods, detached-flow corrections.

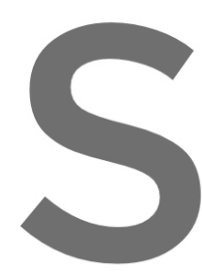

References
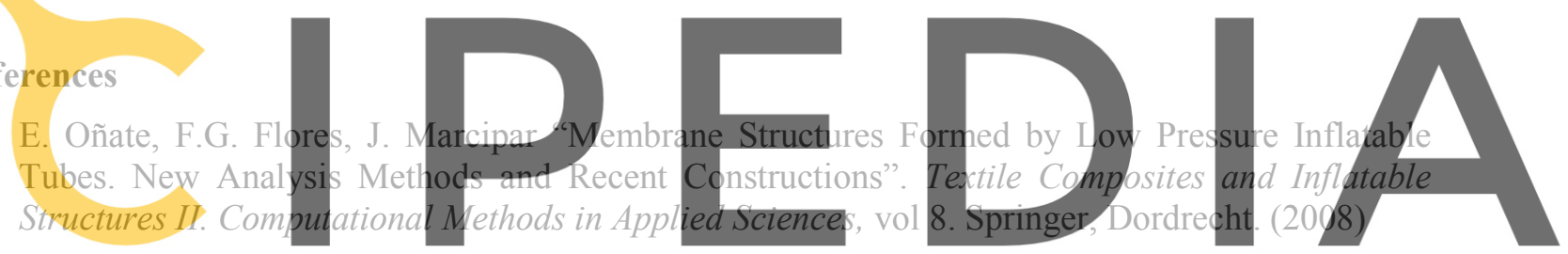

[2] E. Ortega, R. Flores and J. Pons-Prats, "Ram-Air Parachute Simulation with Panel Methods and

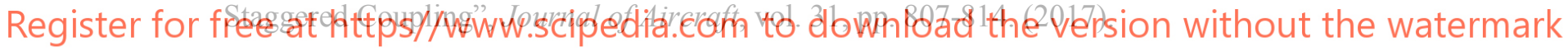

[3] J.M. Gonzalez, J. Marcipar, C. Estruch, E. Cuartero, E. Oñate. "Numerical simulation of an inflated structure for an aircraft hangar" Proceedings of IASS Annual Symposium 2019 -Structural Membranes, Barcelona Spain, (2019)

[4] R. Flores, E. Ortega and E. Oñate, "Simple and efficient numerical tools for the analysis of parachutes," Engineering Computations, vol. 31, issue 5, pp. 957-985. (2014)

[5] R. Flores and E. Ortega "Efficient aerolastic analysis of wind loads on inflatable hangars", Proceedings of IASS Annual Symposium 2019 - Structural Membranes. Barcelona (Spain), (2019).

[6] Ortega, E., Flores, R., Cuartero, E., \& Oñate, EEfficient aeroelastic analysis of inflatable structures using enhanced potential flow aerodynamics. Journal of Fluids and Structures, 90, 230-245 (2019). 